\title{
Hospitalização por doenças infecciosas, parasitismo e evolução nutricional de crianças atendidas em creches públicas
}

\author{
Hospitalization for infectious diseases, parasitism and nutritional \\ evolution of children attended in public child day care centers
}

Dixis Figueroa Pedraza ${ }^{1}$

${ }^{1}$ Programa de Pós-

Graduação em Saúde

Pública, Departamento de

Enfermagem, Universidade

Estadual da Paraíba. Av. das

Baraúnas 351, Bodocongó.

58109-753 Campina

Grande PB Brasil.

dixisfigueroa@gmail.com

\begin{abstract}
The scope of this article is to analyze the influence of hospitalizations by infectious diseases (diarrhea and pneumonia) and parasitism in nutritional evolution, over the period of a year, among children enrolled in municipal public day care centers. Mothers of children in a cohort were asked about previous hospitalization due to diarrhea and pneumonia, as well as in relation to the elimination of worms, during the period of one year of attendance at day care centers. Anthropometrics were measured at each visit. An adjusted model to examine the relationship between infections experienced and growth outcomes (weight and height) was applied. In a subset analysis of 269 children followed up at every visit over the course of 12 months, hospitalization due to pneumonia and parasite infection were associated with decelerated growth in weight and height. Parasitic infection and pneumonia were associated with growth impairment in terms of weight and height. Key words Child day care centers, Infection, Parasites, Pneumonia, Diarrhea
\end{abstract}

Resumo O objetivo deste artigo é analisar a influência das hospitalizações por doenças infecciosas (diarreia e pneumonia) e do parasitismo na evolução nutricional, ao longo de um ano, entre crianças assistidas em creches públicas municipais. Mães de crianças de uma coorte responderam sobre a hospitalização por diarreia e pneumonia, assim como em relação ao parasitismo, durante o período de um ano de frequência à creche. Medidas antropométricas foram tomadas em cada visita. Usou-se modelo ajustado para examinar a relação entre a experiência de processos infecciosos e os desfechos do crescimento (peso e estatura). Numa amostra de 269 crianças monitoradas no período de 12 meses, a hospitalização por pneumonia e a infecção parasitária estiveram associadas com o crescimento desacelerado do peso e da estatura. Infecções parasitárias e pneumonia associaram-se ao comprometimento do crescimento em termos de peso e estatura.

Palavras-chave Creches, Infecção, Parasitos, Pneumonia, Diarreia 


\section{Introdução}

Apesar de que crianças assistidas em creches apresentam maior vulnerabilidade à aquisição de processos infecciosos, os quais podem repercutir negativamente no estado nutricional ${ }^{1-4}$, apontase, também, a assistência a creches como fator protetor do estado nutricional ${ }^{5,6}$. Dessa forma, parece ser que a oferta de alimentos e os cuidados oferecidos nas creches ${ }^{1-4}$ predominam na expressão do estado nutricional. Nessa conjuntura, estabelece-se o reconhecimento da desnutrição como resultado da ingestão alimentar insuficiente e de doenças infecciosas repetidas e graves, fenômeno que pode levar à hospitalização da criança. Assim, desnutrição e processos infecciosos constituem um círculo vicioso no qual a desnutrição aumenta a gravidade e/ou prolonga a duração dos processos de infecções e vice-versa, ou seja, um ciclo no qual a desnutrição é tanto desfecho quanto fator de risco de doenças infecciosas. As condições de vida, do ambiente e o acesso a serviços de saúde permeiam essa relação ${ }^{7-9}$.

Doenças infecciosas, parasitárias e do aparelho respiratório encontram-se entre as principais causas de internação hospitalar em crianças no Brasil. As doenças diarreicas agudas também formam parte desse quadro e têm nas regiões Norte e Nordeste do país as maiores taxas. Esses elementos apresentam-se relevantes na medida em que fundamentam a necessidade de medidas direcionadas à diminuição das internações hospitalares por causas específicas preveníveis. Ainda, a efetividade do sistema de saúde deve considerar a hospitalização como consequência do agravamento de uma condição clínica que não teve atenção de saúde efetiva em momento oportuno ${ }^{9-11}$.

Partindo da hipótese de que as doenças infectocontagiosas influenciam a evolução do estado nutricional, objetivou-se analisar a influência das hospitalizações por doenças infecciosas (diarreia e pneumonia) e do parasitismo na evolução nutricional, ao longo de um ano, entre crianças assistidas em creches públicas municipais.

\section{Métodos}

Este é um estudo de coorte prospectiva integrado ao projeto "Saúde e nutrição das crianças assistidas em creches públicas do município de Campina Grande (PB)". Compreendeu coleta de dados em dois momentos diferentes, com intervalo de 12 meses entre a primeira (outubro a novembro de 2011) e a segunda observação (outubro a no- vembro de 2012). A coleta de dados referiu-se à situação das crianças das creches públicas do município de Campina Grande (PB) pertencentes à Secretaria de Educação.

O município de Campina Grande está localizado no Agreste Paraibano, distante $120 \mathrm{~km}$ de João Pessoa, capital do Estado da Paraíba. O mesmo possui uma área territorial de $671 \mathrm{Km}^{2} \mathrm{e}$ uma população constituída de 402.912 habitantes (estimativa atual). Por dispor de uma posição geográfica privilegiada, Campina Grande é um polo de convergência, com aproximadamente 232 municípios, tanto da Paraíba, quanto de estados vizinhos, cujos habitantes se deslocam para o município em busca dos serviços oferecidos, entre os quais os de saúde.

Ao todo funcionavam, no momento da coleta de dados da primeira observação, 25 creches públicas em bairros distintos do município, situadas em áreas carentes. Segundo a localização, 23 creches estavam na zona urbana e duas na zona rural. Segundo a faixa etária, apenas oito creches apresentavam atendimento em berçário (crianças entre 4 e 20 meses) e 93\% das crianças tinham 24 meses ou mais de idade. O tempo de permanência da criança na creche era integral (8 horas diárias) ou parcial (4 horas diárias), com quantidade de refeições diárias equivalentes.

$\mathrm{Na}$ ocasião, todas as crianças devidamente cadastradas e frequentando as creches consideraram-se elegíveis para participar do estudo, totalizando 2749 crianças, das quais 199 atendidas em berçário. Excluíram-se as crianças gêmeas, adotadas, de mães com idade inferior a 18 anos e aquelas com problemas físicos que dificultassem a avaliação antropométrica, o que gerou 166 exclusões. No caso de crianças irmãs nas creches, uma delas foi sorteada para o estudo.

Baseado no procedimento amostral para descrição da proporção ${ }^{12}$, calculou-se a necessidade de constituir uma amostra representativa de 335 crianças. Considerou-se uma prevalência estima$\mathrm{da}(\mathrm{p})$ de déficit de estatura em crianças menores de cinco anos de $7,0 \%{ }^{13}$, um erro amostral (d) de $3 \%$, e um nível de $95 \%$ de confiança $\left(Z^{2}=\right.$ $\left.1,96^{2}\right)$. O valor calculado $(n=252)$ foi acrescido em $10 \%$ para perdas e recusas, mais efeito de delineamento amostral de 1,2, perfazendo o total de 335. Tamanhos amostrais proporcionais foram considerados para o estudo de crianças segundo a zona de localização da creche (urbana, rural) e a idade da criança (menores de dois anos, dois anos ou mais).

Para a seleção da amostra, nesse primeiro momento, 14 creches foram selecionadas por 
sorteio aleatório simples, sendo uma selecionada entre as localizadas na zona rural e duas entre aquelas com atendimento de berçário. Posteriormente, com posse da lista das crianças assistidas nas creches, foram selecionadas de forma sistemática 15 crianças de 24 meses ou mais por creche de pequeno porte ( 3 creches), 20 por creche de mediano porte ( 3 creches), 25 por creche de grande porte ( 5 creches) e 35 na creche sorteada da zona rural. Em cada uma das duas creches sorteadas com atendimento de berçário, foram selecionadas 35 crianças menores de dois anos.

$\mathrm{Na}$ segunda observação, com o intuito de diminuir as perdas, três tentativas de visitas (uma na creche e duas no domicílio) foram realizadas.
As mães foram estimuladas a participar mediante o desenvolvimento de atividades, como o retorno dos resultados da avaliação do estado nutricional das crianças realizado na primeira visita e ações educativas sobre alimentação, nutrição e doenças infecciosas.

Foram coletadas informações, para o presente estudo, tanto na primeira quanto na segunda observação. Em ambas as ocasiões, as mães responderam um questionário relacionado à situação de saúde e nutrição de seus filhos, previamente testado. Na primeira observação, os questionários foram aplicados nas creches e, na segunda, nas creches ou no domicílio. O Quadro 1 mostra a caracterização das variáveis de estudo.

Quadro 1. Caracterização das variáveis de estudo.

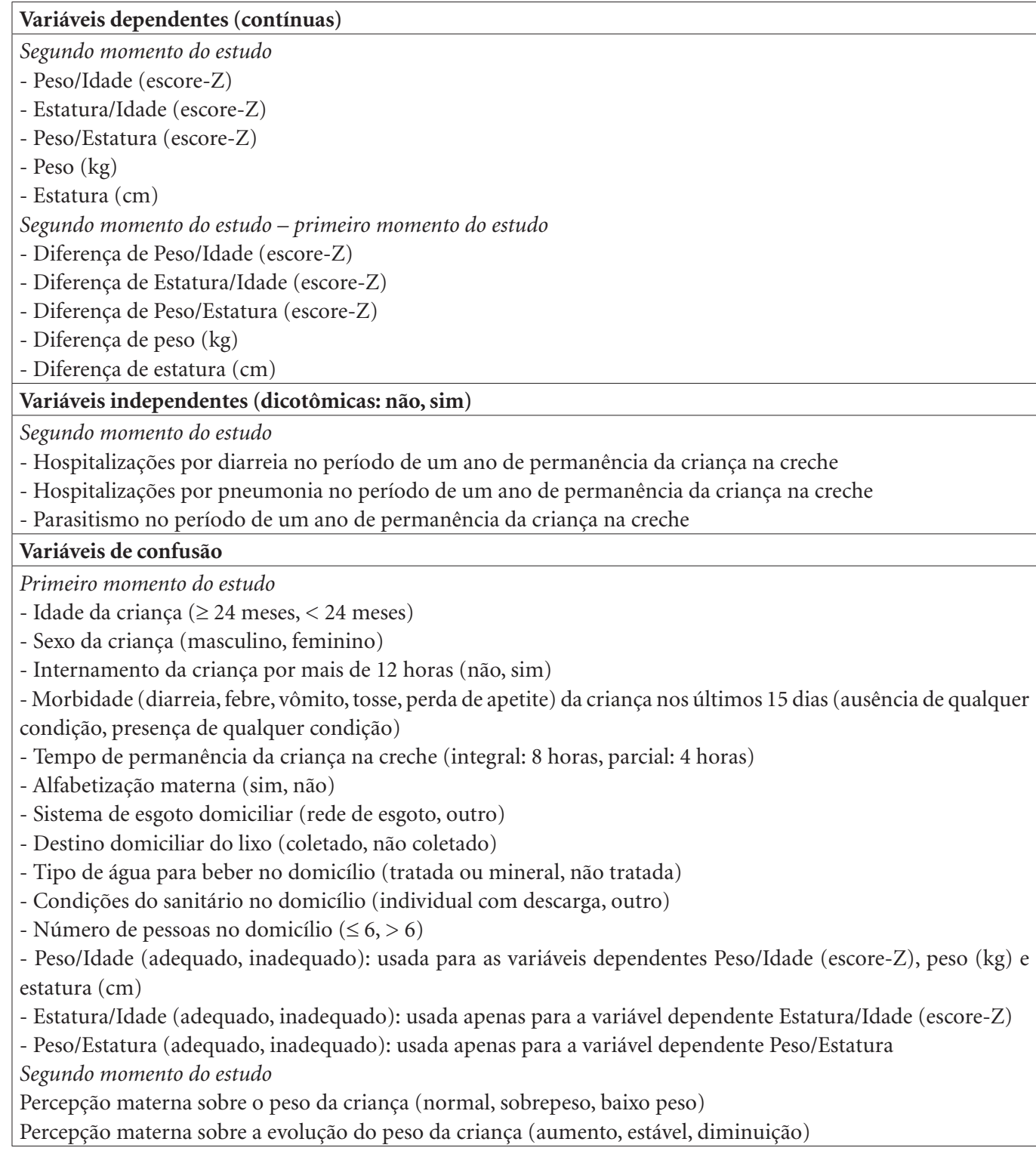


Os dados antropométricos foram obtidos com os mesmos procedimentos e pela mesma equipe nas duas observações. As medições foram realizadas de acordo com normas técnicas padronizadas, obedecendo aos procedimentos recomendados pela $\mathrm{OMS}^{14}$.

As crianças menores de dois anos tiveram o comprimento medido por meio de antropômetro infantil de madeira (Alturexata ${ }^{\circledR}$ ) com amplitude de $130 \mathrm{~cm}$ e subdivisões de $0,1 \mathrm{~cm}$. As crianças de dois anos ou mais tiveram a altura medida por meio de estadiômetro (WCS ${ }^{\circledR}$ ), com amplitude de $200 \mathrm{~cm}$ e subdivisões de $0,1 \mathrm{~cm}$. Todas as crianças foram pesadas em balança eletrônica do tipo plataforma, com capacidade para $150 \mathrm{~kg}$ e graduação em $100 \mathrm{~g}$ (Tanita UM-080 ${ }^{\circledR}$ ). Na pesagem, foi permitida apenas uma peça íntima leve e no caso de crianças que usavam fraldas, estas foram retiradas. O peso de crianças de colo foi calculado pela diferença entre o peso da mãe com a criança no colo e o peso da mãe. As medições foram realizadas, sempre na presença da mãe, em duplicata, aceitando-se variação de $0,3 \mathrm{~cm}$ para medida de estatura e 100 g para medida de peso. Se esses limites fossem eventualmente ultrapassados, repetia-se a mensuração, anotando-se as duas medições com valores mais próximos, utilizando sua média para efeito de registro.

Os escores-Z de Peso/Idade, Estatura/Idade e Peso/Estatura das crianças foram calculados com o programa WHO Anthro 2009. Tomou-se como referência a população do Multicentre Growth Reference Study, atualmente recomendado pela OMS ${ }^{15}$.

Com o objetivo de assegurar a validação da digitação, os dados foram digitados com dupla entrada utilizando-se o programa Excel (Microsoft Inc., Estados Unidos). Após o término da digitação, os dois bancos de dados foram cruzados com a utilização do aplicativo Validate do programa Epi Info v. 6.04b (WHO/CDC, Atlanta, Estados Unidos), possibilitando assim verificar a consistência dos dados e gerando o banco final que foi usado para análise estatística.

O teste $t$ de student foi utilizado para comparar as médias de peso $(\mathrm{kg})$, estatura $(\mathrm{cm})$ e dos escores-Z dos índices antropométricos Peso/Idade, Estatura/Idade e Peso/Estatura nas duas observações. Em análises bivariadas foram estimados modelos de regressão linear generalizados, com função de ligação identidade e distribuição normal das variáveis dependentes, com as variáveis independentes. Em análises multivariadas foram estimados os coeficientes das variáveis independentes ajustados pelas variáveis de confusão. Para cálculo do nível de significância foi aplicado o teste de heterogeneidade de Wald. Todas as conclusões foram tomadas ao nível de significância de $5 \%$. O programa utilizado para as análises estatísticas foi o SPSS v17.0.

O projeto foi aprovado pelo Comitê de Ética em Pesquisa da Universidade Estadual da Paraíba. Todas as mães das crianças que participaram do estudo assinaram o Termo de Consentimento Livre e Esclarecido.

\section{Resultados}

No primeiro momento do estudo, das 335 crianças sorteadas, 13 mães recusaram participar da pesquisa; 14 não compareceram à creche ou não estavam acompanhadas pela mãe no dia da coleta de dados e, em 9, foi impossível realizar a avaliação antropométrica, totalizando o estudo de 299 crianças. No segundo momento, com a perda de 27 crianças, 272 puderam ser reavaliadas (de dois anos ou mais, pois na primeira visita a idade mínima das crianças era de 12 meses). As três crianças que, na ocasião da segunda visita, tinham ultrapassado os cinco anos, foram desconsideradas, totalizando a análise de dados correspondentes a 269 crianças neste estudo.

A Tabela 1 mostra que tanto o peso quanto a estatura das crianças mudou no período de 12 meses de permanência na creche, com variação positiva e significativa. Para os escores-Z de Peso/ Idade, Estatura/Idade e Peso/Estatura não houve variação ao longo do tempo. Segundo os resultados da tabela 2, as hospitalizações por pneumonia estiveram negativamente associadas ao escore- $Z$ de Estatura/Idade após um ano de permanência da criança na creche. As crianças hospitalizadas por pneumonia no último ano tiveram 0,822 escores-Z de Estatura/Idade a menos (IC95\% $-1,567 ;-0,078)$, comparadas às crianças sem antecedentes de hospitalizações por pneumonia.

Enquanto os episódios de hospitalizações não influenciaram as diferenças nos parâmetros antropométricos entre a segunda e a primeira visita (Tabelas 2 e 3), o parasitismo no último ano produziu uma diminuição significativa de 0,211 (IC95\% -0,622; 0,187) no escore-Z de Estatura/ Idade (Tabela 2) e de 0,441kg (IC95\% -0,870; $-0,011$ ) no peso (Tabela 3 ), em relação às crianças sem parasitismo no período. As hospitalizações por pneumonia e o parasitismo foram eventos que influenciaram negativamente tanto o peso $(\mathrm{kg})$ quanto a estatura $(\mathrm{cm})$ das crianças após um ano de permanência das mesmas nas creches (Tabela 3). 
Tabela 1. Evolução do estado nutricional de crianças atendidas em creches públicas municipais de Campina Grande, Paraíba, Brasil, 2011-2012.

\begin{tabular}{lccc}
\hline \multirow{2}{*}{ Variáveis antropométricas } & \multicolumn{3}{c}{ Média (IC95\%) } \\
\cline { 2 - 4 } & Outubro-novembro, 2011 & Outubro-novembro, 2012 & Valor-p $^{*}$ \\
\hline Peso $(\mathrm{kg})$ & $16,68(13,72 ; 19,64)$ & $18,67(15,16 ; 22,18)$ & $<0,001$ \\
Estatura $(\mathrm{cm})$ & $101,04(93,06 ; 109,02)$ & $106,84(98,94 ; 114,74)$ & $<0,001$ \\
Peso/Idade (escore-Z) & $0,096(-0,944 ; 1,136)$ & $0,099(-0,962 ; 1,158)$ & 0,929 \\
Estatura/Idade (escore-Z) & $-0,483(-1,573 ; 0,607)$ & $-0,478(-1,488 ; 0,532)$ & 0,906 \\
Peso/Estatura (escore-Z) & $0,552(-0,448 ; 1,552)$ & $0,572(-0,438 ; 1,582)$ & 0,636 \\
\hline
\end{tabular}

* teste $\mathrm{t}$ de student.

\section{Discussão}

A maioria dos estudos que investigaram determinantes do crescimento infantil, inclusive os direcionados especificamente às crianças que frequentam creches, tem adotado o delineamento transversal, que, por limitação do mesmo, não é capaz de identificar o dinamismo do crescimento das crianças nem a direção das associações ${ }^{5,6}$. Essa limitação estende-se à investigação sobre a associação entre o estado nutricional e o adoecimento por doenças infecciosas nas crianças assistidas em creches, tema pouco abordado na literatura ${ }^{1}$. Nesse sentido, esta investigação disponibiliza, oportunamente, informações longitudinais das variações temporais dinâmicas do estado nutricional antropométrico de crianças, com ênfase na causalidade relacionada a doenças infectocontagiosas.

Os valores médios dos índices antropométricos ao longo dos 12 meses de vida das crianças deste estudo situaram-se nos padrões da eutrofia. No entanto, não foi possível observar diferenças estatísticas relacionadas a esses índices, considerando as mudanças ao longo dos 12 meses de estudo. Apesar de poucos estudos terem desenvolvido análises similares, considerando como população de referência o standard de crescimento estabelecido pela OMS em 2006 ${ }^{14}$, resultados diferentes foram encontrados em outros estudos, nos quais houve variação temporal do estado nutricional favorável ${ }^{16,17}$. Os resultados encontrados neste estudo podem estar circunstanciados às melhorias nas condições de vida da população brasileira, explicativas do declínio da desnutrição no país ${ }^{18,19}$, condicionando à aproximação dos parâmetros antropométricos aos que seriam proporcionados pela plenitude do potencial genético quando superadas as condições adversas do meio ambiente. Esses argumentos apresentam suporte, ainda, considerando que a leitura dos indicadores de peso $(\mathrm{kg})$ e de estatura $(\mathrm{cm})$, ao longo do período do estudo, indica uma realidade na qual as crianças apresentaram aumentos significativos de peso e de estatura.

Ao explorar possíveis variáveis preditoras das médias do índice estatura/idade (escore Z) e da estatura $(\mathrm{cm})$, após um ano de permanência da criança na creche, observou-se estimativa negativa quando a criança foi hospitalizada por pneumonia. Em um estudo de casos e controles realizado na cidade de Pelotas, os autores constataram que as internações por doenças respiratórias agudas estiveram influenciadas pela estatura para idade $^{20}$. Por sua vez, baseado em dados de uma coorte, o comprimento para idade associou-se à maior incidência de hospitalizações por pneumonia aos três meses de vida ${ }^{21}$. Com crianças menores de cinco anos do Estado de Pernambuco, estudo seccional constatou associação entre o internamento por pneumonia e o retardo estatu$\mathrm{ral}^{22}$. Nas análises parece ser importante delimitar o internamento por pneumonia, havendo estudos nos quais não foi possível encontrar a associação quando atentados apenas à informação do internamento ou não, sem prescrever a causa ${ }^{23,24}$. Esse conhecimento é condizente com a concepção da pneumonia como uma das mais graves doenças na infância ${ }^{25}$ e, ainda, importante para o sistema de saúde, principalmente no contexto das doenças por condições sensíveis à atenção primária $^{10,11}$. Assim, constata-se uma literatura consistente, a partir de desenhos epidemiológicos diferentes, que sugere a pneumonia como doença infecciosa que condiciona o crescimento linear das crianças.

Em revisão sistemática da literatura, incluindo artigos de estudos longitudinais desenvolvidos em vários países, constatou-se o efeito negativo da morbidade por diarreia na estatura 
Tabela 2. Análises bruta e ajustada (de acordo com Quadro 1) das variáveis antropométricas (escores-Z e diferenças nos escores-Z de Peso/Idade, Estatura/Idade e Peso/Estatura) no período de um ano de permanência da criança na creche conforme as variáveis independentes. Estudo prospectivo de crianças atendidas em creches públicas municipais, Campina Grande, Paraíba, Brasil, 2011-2012.

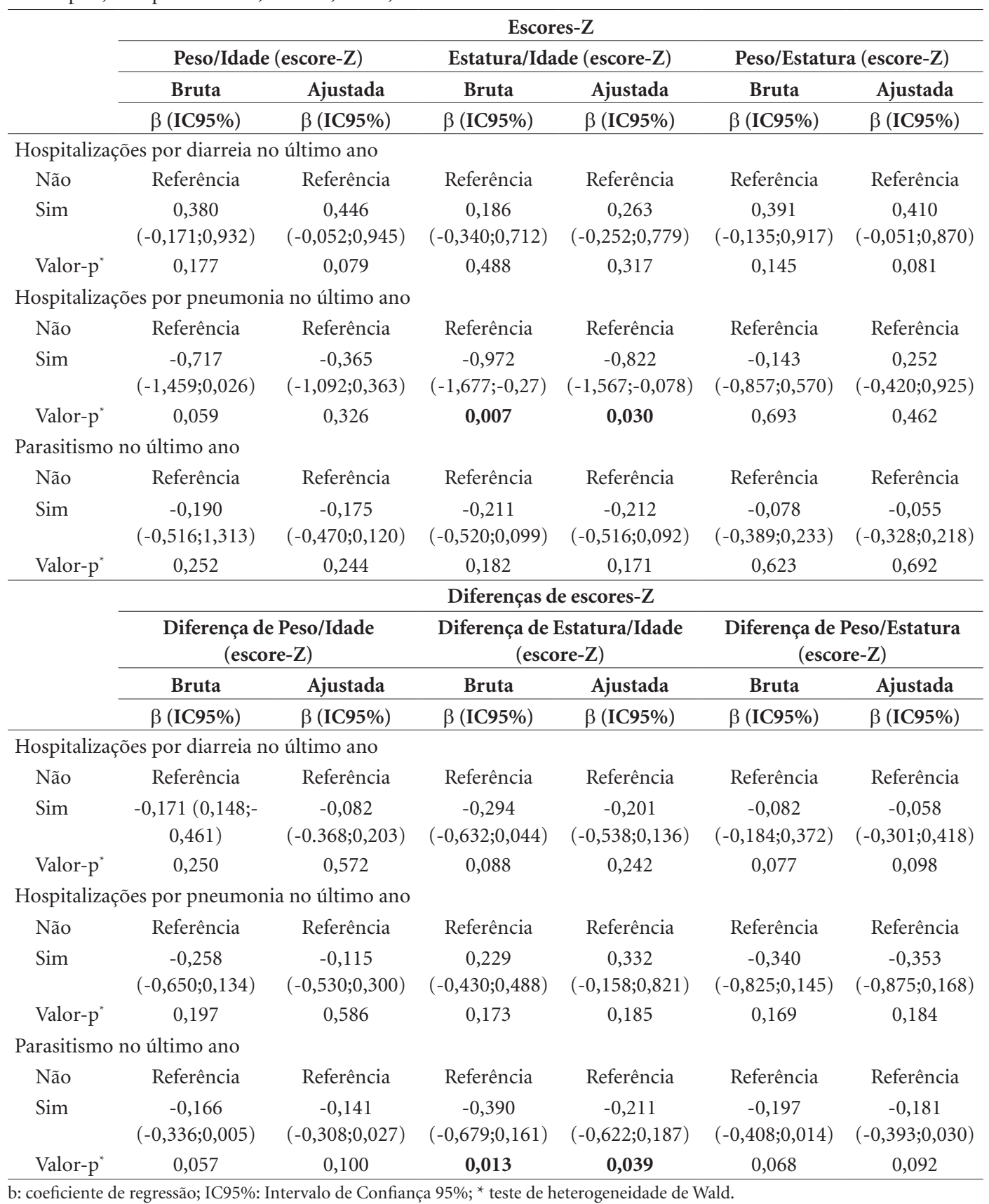

das crianças. No estudo, a chance de déficit de estatura aos 24 meses de idade foi proporcional à quantidade de episódios de diarreia e à quantidade de dias com episódios antes dos 24 meses $^{26}$. Esses resultados são consistentes com a hipótese que associa o risco de déficit de estatura à gravidade dos episódios de diarreia ${ }^{9,26,27}$. Episódios repetidos ou persistentes de diarreia produzem má-absorção de nutrientes ou incapacidade no uso de nutrientes apropriados à manutenção da saúde ${ }^{8}$. Os resultados divergentes do presente estudo podem estar condicionados pela idade das crianças observadas (dois anos ou mais), uma vez que as crianças de idades inferiores são as 
Tabela 3. Análises bruta e ajustada (de acordo com Quadro 1) das variáveis antropométricas (peso, estatura, diferença de peso e diferença de estatura) no período de um ano de permanência da criança na creche conforme as variáveis independentes. Estudo prospectivo de crianças atendidas em creches públicas municipais, Campina Grande, Paraíba, Brasil, 2011-2012.

\begin{tabular}{|c|c|c|c|c|}
\hline & \multicolumn{4}{|c|}{ Peso e Estatura } \\
\hline & \multicolumn{2}{|c|}{ Peso $(k g)$} & \multicolumn{2}{|c|}{ Estatura $(\mathrm{cm})$} \\
\hline & Bruta & Ajustada & Bruta & Ajustada \\
\hline & $\beta($ IC95\%) & $\beta($ IC95\%) & $\beta($ IC95\%) & $\beta($ IC95\%) \\
\hline \multicolumn{5}{|c|}{ Hospitalizações por diarreia no último ano } \\
\hline Não & Referência & Referência & Referência & Referência \\
\hline Sim & $-0,982(-0,669 ; 2,625)$ & $-0,963(-0,707 ; 2,760)$ & $-0,862(-2,718 ; 1,068)$ & $-0,793(-2,059 ; 1,053)$ \\
\hline Valor-p ${ }^{*}$ & 0,238 & 0,264 & 0,376 & 0,332 \\
\hline \multicolumn{5}{|c|}{ Hospitalizações por pneumonia no último ano } \\
\hline Não & Referência & Referência & Referência & Referência \\
\hline Sim & $-0,629(-2,024 ; 0,792)$ & $-0,614(-1,068 ;-0,170)$ & $-0,544(-1,076 ; 0,721)$ & $-0,782(-1,166 ; 0,898)$ \\
\hline Valor- $\mathrm{p}^{*}$ & 0,023 & 0,008 & 0,025 & 0,039 \\
\hline \multicolumn{5}{|c|}{ Parasitismo no último ano } \\
\hline Não & Referência & Referência & Referência & Referência \\
\hline Sim & $-0,489(-1,045 ; 0,083)$ & $-0,414(-0,780 ;-0,011)$ & $\begin{array}{c}-1,933(-2,916 ;- \\
0,941)\end{array}$ & $-0,942(-1,972 ; 0,046)$ \\
\hline \multirow[t]{5}{*}{ Valor-p ${ }^{*}$} & 0,091 & 0,039 & 0,009 & 0,015 \\
\hline & \multicolumn{4}{|c|}{ Diferenças de Peso e de Estatura } \\
\hline & \multicolumn{2}{|c|}{ Diferença de peso $(\mathrm{kg})$} & \multicolumn{2}{|c|}{ Diferença de estatura $(\mathrm{cm})$} \\
\hline & Bruta & Ajustada & Bruta & Ajustada \\
\hline & $\beta($ IC95\%) & $\beta(\mathrm{IC95} \%)$ & $\beta(\mathrm{IC95} \%)$ & $\beta(\mathrm{IC95} \%)$ \\
\hline \multicolumn{5}{|c|}{ Hospitalizações por diarreia no último ano } \\
\hline Não & Referência & Referência & Referência & Referência \\
\hline Sim & $-0,220(-0,990 ; 0,550)$ & $-0,126(-0,707 ; 0,760)$ & $-0,892(-2,182 ; 0,398)$ & $-0,786(-2,077 ; 0,505)$ \\
\hline Valor- $\mathrm{p}^{*}$ & 0,576 & 0,218 & 0,175 & 0,233 \\
\hline \multicolumn{5}{|c|}{ Hospitalizações por pneumonia no último ano } \\
\hline Não & Referência & Referência & Referência & Referência \\
\hline Sim & $-0,909(-1,944 ; 0,127)$ & $-0,574(-1638 ; 0,490)$ & $-1,111(-2,247 ; 0,015)$ & $-0,427(-1,093 ; 0,186)$ \\
\hline Valor- $\mathrm{p}^{*}$ & 0,085 & 0,291 & 0,072 & 0,199 \\
\hline \multicolumn{5}{|c|}{ Parasitismo no último ano } \\
\hline Não & Referência & Referência & Referência & Referência \\
\hline Sim & $-0,494(-0,945 ;-0,043)$ & $-0,441(-0,870 ;-0,011)$ & $-0,343(-1,106 ; 0,419)$ & $-0,294(-1,057 ; 0,469)$ \\
\hline Valor- $\mathrm{p}^{*}$ & 0,032 & 0,044 & 0,377 & 0,450 \\
\hline
\end{tabular}

de maior suscetibilidade ${ }^{9,27}$. Entretanto, estudo seccional desenvolvido com crianças menores de cinco anos do Estado de Pernambuco constatou associação entre o internamento por diarreia e o retardo estatural ${ }^{22}$. Por sua vez, a associação também não foi verificada em outras localidades do país ${ }^{9,27}$. A divergência entre esses achados pode advir das representações próprias para cada tempo e espaço dos fatores de risco, estabelecendo-se melhorias nos indicadores de saúde e nutrição das crianças brasileiras marcadas pela desigual- dade, com diminuição acentuada na incidência da diarreia e dos seus desfechos (hospitalização e mortalidade infantil) inclusive na região Nordeste es-30. $^{20}$.

Segundo os resultados apresentados, a referência materna ao parasitismo apresentou associação com indicadores de peso e de estatura, o que se sugere devido ao aparecimento ou agravamento da desnutrição associado a mecanismos como lesão da mucosa, exsudação intestinal, má -absorção de micronutrientes, alteração do me- 
tabolismo de sais biliares, competição alimentar, diminuição do apetite, favorecimento da proliferação bacteriana e hemorragias ${ }^{31,32}$. Esses resultados condizem com os de outros estudos, inclusive longitudinais, que têm reportado a influência das infecções parasitárias nos indicadores antropométricos de peso e estatura das crianças ${ }^{33-37}$.

O fato de, neste estudo, o crescimento ponderal e de peso para a idade no período de um ano de frequência das crianças à creche ter sido influenciado apenas pelo adoecimento por parasitose, e não por diarreia e pneumonia, pode ter explicações relacionadas com as maiores taxas de morbidade das parasitoses e sua associação tanto com diarreia crônica quanto com pneumonia ${ }^{1,31,38}$. Além disso, sinaliza-se o controle da desnutrição aguda e global entre as crianças brasileiras, representando, assim, o déficit de estatura o principal signo da desnutrição no país ${ }^{12,28}$, pressuposto que pode, ainda, ter influenciado a ausência de efeitos da hospitalização por diarreia e por pneumonia nesses indicadores. Não obstante, algumas pesquisas têm constatado a perda ponderal associada ao adoecimento/internação por pneumonia ${ }^{20,21,39}$.

No que concerne à população específica deste estudo, a relevância dos resultados sobressai, considerando que a convivência coletiva e aglomerada das crianças em creches possibilita a transmissão de doenças infecciosas ${ }^{1}$. A partilha de ambientes aumenta o risco de pneumonia por causa da facilidade de transmissão de agentes patogênicos através da respiração, bem como o risco de doenças infecciosas causadoras de diarreia, acrescentando riscos às incidências das principais causas de hospitalização na infância (pneumonia e diarreia $)^{1,20,21}$. Por sua vez, as condições inadequadas de higiene, de saneamento básico e de educação sanitária facilitam a disseminação de doenças parasitárias ${ }^{1,32,40}$. O risco de parasitoses intestinais é particularmente alto entre as crianças do Nordeste, uma das regiões mais pobres e populosas do Brasil tropical ${ }^{40}$. A transmissão feco -oral representa, ainda, uma maior vulnerabilidade das crianças institucionalizadas em creches para alguns parasitas ${ }^{38}$. Na conjuntura anterior, a disponibilidade de água e as condições higiênico-sanitárias constituem importantes fatores na prevenção da desnutrição ao ter impactos diretos no desenvolvimento de doenças infecciosas, particularmente a diarreia ${ }^{8}$.

Por fim, deve-se enfatizar que o estado nutricional é diretamente influenciado pela infecção, mas, constitui, ao mesmo tempo, um dos principais moduladores da resposta imune, e, portanto, é importante determinante do risco de doenças infecciosas ${ }^{41}$. Assim, este padrão de interação sinérgico entre infecção e estado nutricional induz à coexistência e influência mútua desses dois agravos, tornando difícil identificar o que é causa e o que é efeito ${ }^{1}$. Esse fenômeno é crucial à compreensão da dinâmica populacional das infecções, é particularmente importante nas crianças assistidas em creches, por sua maior vulnerabilidade, e alerta para os benefícios advindos da adoção de medidas preventivas conjuntas ${ }^{1,41}$.

\section{Conclusão}

Os resultados sugerem o comprometimento do crescimento em termos de peso e estatura, devido a processos parasitários e à hospitalização por pneumonia. 


\section{Referências}

1. Pedraza DF, Queiroz D, Sales MC. Doenças infecciosas em crianças pré-escolares brasileiras assistidas em creches. Cien Saude Colet 2014; 19(2):501-518.

2. Oliveira JS, Lira PIC, Carvalho AGC, Barros MFA, Lima MC. Fatores associados ao estado nutricional em crianças de creches públicas do município de Recife, PE, Brasil. Rev Bras Epidemiol 2013; 16(2):502-512.

3. Goulart RMM, Banduk MLS, Taddei JAAC. Uma revisão das ações de nutrição e do papel do nutricionista em creches. Rev Nutr 2010; 23(4):655-665.

4. Biscegli TS, Romera J, Candido AB, Santos JM, Candido ECA, Binotto AL. Estado nutricional e prevalência de enteroparasitoses em crianças matriculadas em creche. Rev Paul Pediatr 2009; 27(3):289-295.

5. Pereira AS, Lanzillotti HS, Soares EA. Frequência à creche e estado nutricional de pré-escolares: uma revisão sistemática. Rev Paul Pediatr 2010; 28(4):366-372.

6. Figueroa Pedraza D, Souza MM, Rocha ACD. Fatores associados ao estado nutricional de crianças pré-escolares brasileiras assistidas em creches públicas: uma revisão sistemática. Rev Nutr 2015; 28(4):451-463.

7. Khan Y, Bhutta ZA. Nutritional deficiencies in the developing world: current status and opportunities for intervention. Pediatr Clin North Am 2010; 57(6):14091441.

8. Imdad A, Sadig K, Bhutta ZA. Evidence-based prevention of childhood malnutrition. Curr Opin Clin Nutr Metab Care 2011; 14(3):276-285.

9. Rocha MCGS, Carminate DG, Tibiriçá SHC, Carvalho IP, Silva MLR, Chebli JMF. Acute diarrhea in hospitalized children of the municipality of Juiz de Fora, MG, Brazil: prevalence and risk factors associated with disease severity. Arq Gastroenterol 2012; 49(4):259-265.

10. Oliveira BRG, Viera CS, Collet N, Lima RAG. Causas de hospitalização no SUS de crianças de zero a quatro anos no Brasil. Rev Bras Epidemiol 2010; 13(2):268-277.

11. Moura BLA, Cunha RC, Aquino R, Medina MG, Mota ELA, Macinko J, Dourado I. Principais causas de internação por condições sensíveis à atenção primária no Brasil: uma análise por faixa etária e região. Rev Bras Saude Matern Infant 2010; 10(Supl. 1):S83-S91.

12. Figueroa Pedraza D, Rocha ACD, Sousa CPC. Crescimento e deficiências de micronutrientes: perfil das crianças assistidas no núcleo de creches do governo da Paraíba, Brasil. Cien Saude Colet 2013; 18(11):33793390.

13. Monteiro CA, Conde WL, Konno SC, Lima AL, Silva AC, Benicio MH. Avaliação antropométrica do estado nutricional de mulheres em idade fértil e crianças menores de cinco anos. In: Brasil. Ministério da Saúde (MS). Pesquisa Nacional de Demografia e Saúde da Criança e da Mulher: PNDS 2006: Dimensões do processo reprodutivo e da saúde da criança. Brasília: MS; 2009. p. 213-230.

14. World Health Organization (WHO). Physical status: the use and interpretation of anthropometry. Geneva: WHO; 1995. (WHO - Technical Report Series, 854).

15. World Health Organization (WHO). WHO Child Growth Standards. Length/height-for-age, weight-forage, weight-for-length, weight-for-height and body mass index-for-age. Methods and development. Geneva: WHO; 2006.
16. Barros AJD, Victora CG, Santos IS, Matijasevich A, Araújo CL, Barros FC. Infant malnutrition and obesity in three population-based birth cohort studies in Southern Brazil: trends and differences. Cad Saude Publica 2008; 24(Supl. 3):S417-S4S26.

17. Pereira AS, Peixoto NGA, Nogueira Neto JF, Lanzillotti HS, Soares EA. Estado nutricional de pré-escolares de creche pública: um estudo longitudinal. Cad Saúde Colet 2013; 21(2):140-147.

18. Monteiro CA, Benicio MHD'A, Conde WL, Konno SC, Lima ALL, Barros AJD, Victora CG. Desigualdades socioeconômicas na baixa estatura infantil: a experiência brasileira, 1974-2007. Estud Av 2013; 27(78):36-49.

19. Pedraza DF. Crescimento linear das crianças brasileiras: reflexões no contexto da equidade social. Rev Nut 2016; 29(2):287-296.

20. Macedo S, Baptista AM, Albernaz E, Post P, Knorst M. Fatores de risco para internação por doença respiratória aguda em crianças até um ano de idade. Rev Saude Publica 2007; 41(3):351-358.

21. Ngale KMA, Santos IS, González-Chica DA, Barros AJD, Matijasevich A. Bed-sharing and risk of hospitalisation due to pneumonia and diarrhoea in infancy: the 2004 Pelotas Birth Cohort. J Epidemiol Community Health 2013; 67:245-249.

22. Rissin A, Figueiroa JN, Benício MHD'A, Batista Filho M. Retardo estatural em menores de cinco anos: um estudo "baseline". Cien Saude Colet 2011; 16(10):40674076.

23. Queiroz VAO, Assis AMO, Pinheiro SMC, Ribeiro Junior HC. Preditores do crescimento linear no primeiro ano de vida em uma coorte prospectiva de crianças a termo com peso adequado. J Pediat 2012; 88(1):79-86.

24. Ramalho AA, Mantovani SAS, Delfino BM, Pereira TM, Martins AC, Oliart-Guzmán H, Brãna AM, Branco FL, Campos RG, Guimarães AS, Araújo TS, Oliveira CS, Codeço CT, Muniz PT, da Silva-Nunes M1.Nutritional status of children under 5 years of age in the Brazilian Western Amazon before and after the Interoceanic highway paving: a population-based study. $B M C$ Public Health 2013; 13:1098.

25. Paranhos VD, Pina JC, Mello DF. Atenção integrada às doenças prevalentes na infância e o enfoque nos cuidadores: revisão integrativa da literatura. Rev Latino-Am Enfermagem 2011; 19(1):203-211.

26. Checkley W, Buckley G, Gilman RH, Assis AMO, Guerrant RL, Morris SS, Mølbak K, Valentiner-Branth P, Lanata CF, Black RE; Childhood Malnutrition and Infection Network. Multi-country analysis of the effects of diarrhoea on childhood stunting. Int Journ Epidem 2008; 37(4):816-830.

27. Costa ADPV, Silva GAP. Indicação de terapia de reidratação oral no setor de emergência: decisão baseada na clínica? Rev Paul Pediatr 2010; 28(2):215-220.

28. Pedraza DF. Preditores de riscos nutricionais de crianças assistidas em creches em município de porte médio do Brasil. Cad Saúde Coletiva 2017; 25(1):14-23.

29. Victora CG, Aquino EML, Leal MC, Monteiro CA, Barros FC, Szwarcwald CL. Saúde de mães e crianças no Brasil: progressos e desafios. Lancet 2011; (Supl. Saúde no Brasil):32-46. 
30. Barreto ML, Teixeira MG, Bastos FI, Ximenes RAA, Barata RB, Rodrigues LC. Sucessos e fracassos no controle de doenças infecciosas no Brasil: o contexto social e ambiental, políticas, intervenções e necessidades de pesquisas. Lancet 2011; (Supl. Saúde no Brasil):47-60.

31. Andrade EC, Leite ICG, Rodrigues VO, Cesca MG. Parasitoses intestinais: uma revisão sobre seus aspectos sociais, epidemiológicos, clínicos e terapêuticos. Rev APS 2010; 13(2):231-240.

32. Bryan P, Mejia R. Invited Commentary on Growth and Development in Children with Gastrointestinal Parasitic Infections. Curr Trop Med Rep 2015; 2(4):233-237.

33. Gyorkos TW, Maheu-Giroux M, Casapia M, Joseph SA, Creed-Kanashiro H. Stunting and helminth infection in early preschool-age children in a resource-poor community in the Amazon lowlands of Peru. Transactions of the Royal Society of Tropical Medicine and Hygiene 2011; 105:204-208.

34. LeBeaud AD, Singer MN, McKibben M, Mungal $P$, Muchiri EM, McKibben E, Gildengorin G, Sutherland LJ, King CH, King CL, Malhotra I.. Parasitism in children aged three years and under: relationship between infection and growth in rural coastal Kenya. PLoS Negl Trop Dis 2015; 9(5):e0003721.

35. Botero-Garces JH, Garcia-Montoya GM, Grisales-Patino D, Aguirre-Acevedo DC, Alvarez-Uribe MC. Giardia intestinalis and nutritional status in children participating in the complementary nutrition program, Antioquia, Columbia, May to October 2006. Rev Inst Med Trop Sao Paulo 2009; 51(3):155-162.

36. Molloy SF, Tanner CJ, Kirwan P, Asaolu SO, Smith HV, Nichols RAB, Connelly L, Holland CV.Sporadic Cryptosporidium infection in Nigerian children: risk factors with species identification. Epidemiol Infect 2011; 139(6):946-954.
37. Ajjampur SSR, Koshy B, Venkataramani M, Sarkar R, Joseph AA, Jacob KS, Ward H, Kang G. Effect of Cryptosporidial and giardial diarrhea on social maturity, intelligence and physical growth in children in a semi-urban slum in south India. Ann Trop Paediatr 2011; 31(3):205-212.

38. Braz AS, Andrade CAF, Mota LMH, Lima CMBL. Recomendações da Sociedade Brasileira de Reumatologia sobre diagnóstico e tratamento das parasitoses intestinais em pacientes com doenças reumáticas autoimunes. Rev Bras Reumatol 2015; 55(4):368-380.

39. Rocha GA, Rocha EJM, Martins CV. Hospitalização: efeito sobre o estado nutricional em crianças. J Pediatr 2006; 82(1):70-74.

40. Lander LR, Lander AG, Houghton L, Williams SM, Costa-Ribeiro H, Barreto DL, Mattos AP,Gibson RS. Factors influencing growth and intestinal parasitic infections in preschoolers attending philanthropic daycare centers in Salvador, Northeast Region of Brazil. Cad Saude Publica 2012; 28(11):2177-2188.

41. Werneck GL, Hasselmann MH, Gouvê TG. Panorama dos estudos sobre nutrição e doenças negligenciadas no Brasil. Cien Saude Colet 2011;16(1):39-62.

Artigo apresentado em 05/01/2016

Aprovado em 04/05/2016

Versão final apresentada em 06/05/2016 